\title{
Research Progress of the Intervention Effect of Mindfulness Therapy on Patients with Hypertension
}

\author{
Yi Liu ${ }^{1}$, Jian-quan Liu ${ }^{2}$, Xiang Li ${ }^{3}$, Zhi-bing Zhong,"* \\ ${ }^{1}$ College of Graduate, Jiangxi University of Traditional Chinese Medicine, Nanchang, Jiangxi 330004, China \\ ${ }^{2}$ College of Graduate, Jiangxi University of Traditional Chinese Medicine, Nanchang, Jiangxi 330004, China \\ ${ }^{3}$ College of Graduate, Jiangxi University of Traditional Chinese Medicine, Nanchang, Jiangxi 330004, China \\ ${ }^{4}$ Psychological Counseling Center, Jiangxi University of Traditional Chinese Medicine, Nanchang, Jiangxi 330004, \\ China \\ *Corresponding author. Email:494234679@qq.com
}

\begin{abstract}
Nowadays, the prevalence of hypertension in China is still on the rise. For patients with hypertension, the combination of drug therapy and adjuvant therapy is usually used. Mindfulness Therapy as a non-medicine psychosomatic, has achieved good results in clinical application, and has become the mainstream psychological intervention technology in the treatment of chronic diseases. Mindfulness therapy plays a huge role in alleviating the negative emotions of hypertension patients, improving symptoms and self-management. This article will discuss the overview of mindfulness, mindfulness intervention technology and its application in hypertension patients, in order to provide basis for the application of this psychological intervention method in the nursing and clinical work of patients with hypertension.
\end{abstract}

Keywords: social sciences, Mindfulness therapy, hypertension, review

\section{MINDFULNESS}

Mindfulness originated in the East and developed into a scientific training method beneficial to human beings in the West. Western psychologists have refined the core idea of mindfulness in Buddhism, which is separated from religious elements. Today's mindfulness in psychology and clinical application does not involve any religious beliefs, rituals and behaviors.

Mindfulness training is a kind of practice that emphasizes guiding participants to pay attention to the present and the place, improving self-awareness, improving concentration and self-management ability. At present, many scholars are very interested in mindfulness. There are more and more researches on mindfulness, and the application scope of mindfulness is becoming wider and wider. The research of different students shows that mindfulness training has obvious effects on improving happiness, resisting negative emotions, offsetting loneliness and improving individual mental health. Compared with the traditional psychological intervention practice, mindfulness based interventions (MBIs) has its unique side. It emphasizes "focusing on the present" in a non-critical way, and does not involve religious thoughts and rituals, so mindfulness does not belong to a specific culture at all. A series of meditation and psychological intervention therapies centered on mindfulness are called MBIs [1]. Traditional mindfulness therapy includes mindfulness based stress reduction (MBSR), mindfulness based cognitive therapy (MBCT), and dialectical behavior therapy (DBT). However, modern psychology has different views on the concept of mindfulness therapy. It thinks that mindfulness therapy includes MBSR, MBCT, mindfulness Meditations (MMS), acceptance of reality therapy and meditation.

\section{HYPERTENSION}

Hypertension is one of the most common psychological factors, which plays an important role in its occurrence, development and prognosis. It is also the main culprit of cardiovascular disease. At the same time, hypertension can also lead to the occurrence of some other chronic diseases. Hypertension mostly occurs in the elderly patients, but in recent years, with the improvement of people's quality of life, the group of hypertension patients is becoming younger. High blood pressure will bring double burden to people's body and mind, and high treatment cost will bring great pressure to patients' psychology.

At present, the prevention and treatment of hypertension in China is more inclined to drug treatment, while there are few studies on the psychological aspects to consider the causes and treatment of hypertension. From the psychological point of view to think about the causes of hypertension, to find the intervention methods of hypertension, can fundamentally solve the economic pressure of hypertension patients, but also can reduce the side effects of drugs on patients. 


\section{TECHNIQUES OF MINDFULNESS THERAPY}

MBIs can be divided into formal practice and informal practice [2]. Formal practice of MBIs mainly involves mindfulness meditation, mindfulness breathing, mindfulness yoga and body scanning. Informal practice can detect happy and unpleasant events. Hypertension patients have certain requirements for all aspects of physical quality when they do mindfulness yoga exercises, so in general, hypertension patients use the following three ways to carry out MBIs exercises: mindfulness meditation, mindfulness breathing and body scanning. Meditation is the most basic, core and main technology of MBIs.

\section{DATA SOURCES AND METHODS}

The data sources are CNKI (China journal full text database, China doctoral dissertation database, China important conference thesis full text database and China important newspaper full text database) and MEDLINE (including bibliographic information of articles from medical journals, medicine, nursing, pharmacy, dentistry, veterinary medicine and health care) databases. The content samples are from February 2010 to now. The inclusion criteria were as follows: 1. Study design type: randomized controlled trial, single group pre-test and multi baseline design; 2. Subjects: patients who met the diagnostic criteria of hypertension, patients older than 18 years old, excluded from other serious physical diseases and mental disorders; 3. Intervention measures: all mindfulness based psychotherapy, including MBSR, MBCT, DBT, MMS, was included in the study ; 4. Outcome indicators: clinical blood pressure, namely systolic and diastolic blood pressure, psychological indicators for anxiety and depression, and sleep quality.

\section{EFFECT OF MINDFULNESS ON HYPERTENSION AND ITS COMPLICATIONS}

Through consulting the intervention literature of mindfulness on hypertension patients, it is found that mindfulness has a good intervention effect on hypertension and all kinds of adverse symptoms caused by hypertension. When people's emotions are unstable, the excitability of the cerebral cortex and hypothalamus is very high, which produces adrenaline and angiotensin, which promotes vasospasm and blood pressure. Hypertension can lead to heart attack or sudden stroke. Although antihypertensive drugs can improve the risk of heart disease, one third of patients with hypertension can not be effectively controlled. There are many studies that show that mindfulness meditation is good for lowering blood pressure. Mindfulness requires the patient to focus on the moment with an "acceptance" and "non-criticism" mentality, thereby regulating the patient's emotional state.
When the patient's emotion is stable, the somatization symptoms will be relieved, and the systolic and diastolic blood pressure will also be reduced. Some studies have pointed out that the heart rate of hypertensive patients without complications should be controlled within 60-80 times per minute. When they have cardiovascular and encephalopathy complications, the heart rate should be controlled within 50-65 times per minute [3]. Too fast or too slow heart rate may promote the occurrence of cardiovascular extreme events. Therefore, in the nursing of hypertension patients, we should not only pay attention to the change of blood pressure, but also control the resting heart rate of hypertension patients within 70 times per minute. Mindfulness decompression therapy can improve the immune function of the body and regulate the psychology by increasing the density of gray matter in hippocampus and changing the activity of frontal lobe. Therefore, it can effectively reduce stress and pain, improve sleep quality, relieve negative emotions and improve self-efficacy, patients can train a good and stable mentality through mindfulness training.

\subsection{Effect of Mindfulness Intervention on Blood Pressure}

Nejati et al. [4] showed that MBSR had a significant effect on hypertension. The study of Palla [5] shows that MBSR has a significant effect of reducing blood pressure (systolic blood pressure decreased by $21.92 \mathrm{mmhg}$, diastolic blood pressure decreased by $16.7 \mathrm{mmHg}$ ). Hughes et al. [6] found that MBSR has more significant antihypertensive effect than progressive muscle relaxation (PMR). Abbott et al. [7] found that MBSR has a moderate degree of hypotensive effect on patients with hypertension, and the blood pressure of patients with high baseline blood pressure or combination drugs decreased more significantly. Wolff et al. [8] found that MBSR for 12 weeks had significant effect on reducing diastolic pressure, but not on systolic pressure. Park [9] found that MBSR has a significant antihypertensive effect on hypertensive patients with chronic kidney disease. Ponte [10] found that after 8 weeks of MBSR training, the 24-hour ambulatory blood pressure night systolic blood pressure score in the intervention group was significantly lower than that in the control group. The follow-up after 20 weeks showed that there was no significant difference in blood pressure between the two groups, but in terms of anxiety and depression, the intervention group was significantly lower than the control group. According to a meta analysis based on RCT, the systolic and diastolic blood pressure of the intervention group were significantly lower than that of the control group when MBSR was used in the clinical clock, but the results were inconsistent with the former when ambulatory blood pressure monitoring was conducted. [11] In conclusion, mindfulness therapy has a significant effect on reducing blood pressure (including systolic and diastolic blood pressure) in patients with hypertension. 


\subsection{Effect of Mindfulness Intervention on Sleep of Patients with Hypertension}

Mindfulness decompression therapy can improve the sleep status, cognitive level, negative emotions and quality of life of hypertensive patients. Studies by Gu Xiaoyan et al. [12] showed that after 8 interventions with MBIs, the observation group had significantly lower subjective sleep quality, time to fall asleep, sleep time, sleep efficiency, daytime dysfunction and overall sleep quality compared with the control group 2. The results show that MBIs intervention has a positive effect on improving the sleep quality of hypertensive patients. Giving patients individualized psychological adjustment and family health guidance and other nursing models can help improve the sleep quality of hypertensive patients. Studies by Zhang Jiwei et al. [13] showed that patients in the observation group were better than the control group in terms of subjective sleep quality, time to fall asleep, sleep time, sleep efficiency, daytime dysfunction and total sleep quality scores after MBIs intervention for ten days. In summary, MBIs have a significant effect on improving the sleep quality of hypertensive patients.

\subsection{Effect of Mindfulness Intervention on Anxiety and Depression Symptoms of Hypertensive Patients}

The research of Liu Zhihui et al. [14] showed that Puerarin Combined with mindfulness meditation has a good therapeutic effect on the anxiety and depression of patients with hypertension, and has a significant alleviation effect on clinical symptoms such as depression and anxiety, with less adverse reactions. Puerarin can dilate blood vessels and inhibit platelet clotting, so it can effectively relieve hypertension. Mindfulness meditation has a good intervention effect on anxiety and depression symptoms. Therefore, in the influence of mindfulness intervention on anxiety and depression symptoms of patients with hypertension, whether hypertension plays an intermediary role is worth exploring. Miao su et al. [15] measured the level of anxiety and depression in elderly patients with hypertension. The results showed that the level of anxiety and depression in MBIs intervention group was significantly lower than that in the control group. Qin Bing [16] used MBIs combined with acupoint massage for patients with essential hypertension. The study found that the anxiety state of the experimental group was effectively relieved and the rehabilitation process of the patients was accelerated. Goldin and gross [17] and other studies show that MBIs can effectively alleviate anxiety, effectively improve the individual's emotional regulation ability, and maintain emotional stability. The improvement of mindfulness level is an important factor of emotional relief caused by intervention. [18].

\section{CONCLUSION AND PROSPECT}

At present, MBIs has only auxiliary effect on the treatment of hypertension. The current mainstream treatment in China is drug therapy. However, MBIs has been involved in the treatment of hypertension, and mindfulness therapy has a considerable acceptance in the population, because mindfulness therapy has the characteristics of simple operation, economic and practical. MBIs plays an important role in regulating blood pressure, improving sleep quality, relieving anxiety and depression symptoms, and reducing medical costs. At present, there are too few samples to intervene hypertension patients with MBIs, and the operation methods of MBIs are different. The tracking effect of MBIs on reducing blood pressure also needs to be further verified in the follow-up study. Based on the above discussion, more samples and more rigorous operation procedures are needed to verify the hypotensive effect of mindfulness therapy on patients in the future research, so as to promote the psychotherapy process of hypertension, a physical and mental disease, and enrich the treatment methods of chronic diseases at the world difficult level such as cardio cerebrovascular diseases.

Most of the research results of mindfulness decompression therapy are exciting, but sometimes there are some unsatisfactory aspects. First of all, most of the literature research adopts the research design of pre-test and post-test. The lack of comparison of the control group makes it difficult to exclude the influence of many factors such as demand characteristics and placebo effect, thus affecting the reliability of the experiment. For example, Goldin and Gross [17] used the pre-test and post-test experimental design to train anxiety patients with an average age of 35.2 years old during the study of anxiety patients. Because the experimental design lacked a control group during the training procession therapy, some non-specific factors, such as social expectations, the social support of the subjects, and the attention of the therapist, may become factors that affect the experimental results. Secondly, the sample size of the subjects in the study of mindfulness decompression therapy is usually relatively small, and the lack of sample size may affect the validity of the experiment. Therefore, future research should expand the sample size, or expand the sample area for confirmatory research, in order to enhance the validity of experimental research. Thirdly, the current research on the effect of mindfulness decompression therapy on hypertensive patients is generally limited to patients with grade 1 , and there are few studies at other levels. In the future research, we can expand the scope of research objects, and make mindfulness decompression therapy become an effective method of universal treatment and health care. In addition, those who cannot accept mindfulness meditation or have religious taboos should be excluded from the scope of the research object or clinical application. 


\section{ACKNOWLEDGMENT}

This work was supported by Jiangxi provincial education and science "13th five-year plan" 2020 annual project(No.146) and Research on College Students' Leadership Training under the Double-Education Mode (2018jzzd-1).

\section{REFERENCES}

[1] Wang Yunxia and Jiang Chunlei, biological mechanism and physical and mental health of mindfulness meditation. Chinese Journal of mental health, 2016.30 (02): 105-108

[2] Sheng Yuanyuan, Liu Huijun and he Xinji, clinical application of mindfulness intervention in cancer rehabilitation. Progress in psychological science, 2017.25 (12): 2124-2135

[3] Guo Yifang, should heart rate be the target of drug intervention for hypertensive patients? Chinese Journal of hypertension, 2017.25 (08): 709-713

[4] Nejati, S., et al., Effect of Group Mindfulness-Based Stress-Reduction Program and Conscious Yoga on Lifestyle, Coping Strategies, and Systolic and Diastolic Blood Pressures in Patients with Hypertension. J Tehran Heart Cent, 2015. 10(3): p. 140-8.

[5] Palta, P., et al., Evaluation of a mindfulness-based intervention program to decrease blood pressure in low-income African-American older adults. J Urban Health, 2012. 89(2): p. 308-16.

[6] Hughes, J.W., et al., Randomized controlled trial of mindfulness-based stress reduction for prehypertension. Psychosom Med, 2013. 75(8): p. 721-8.

[7] Abbott, R.A.W.R., Effectiveness of mindfulness-based stress reduction and mindfulness based cognitive therapy in vascular disease: A systematic review and meta-analysis of randomised controlled trials. Journal of psychosomatic research, 2014. 5(76).

[8] Wolff M, S.K.L.S., Impact of yoga on blood pressure and quality of life in patients with hypertension-a controlled trial in primary care, matched for systolic blood pressure. BMC cardiovascular disorders, 2013. 13(1): p. 111.

[9] Park, J., R.H. Lyles and S. Bauer-Wu, Mindfulness meditation lowers muscle sympathetic nerve activity and blood pressure in African-American males with chronic kidney disease. American Journal of Physiology-Regulatory, Integrative and Comparative Physiology, 2014. 307(1): p. R93-R101.
[10] Ponte Márquez P H, F.A.S.M., R.B.P.A. Human and Hypertension.

[11] Solano Lopez, A.L., Effectiveness of the Mindfulness-Based Stress Reduction Program on Blood Pressure: A Systematic Review of Literature. Worldviews on Evidence-Based Nursing, 2018. 15(5): p. 344-352.

[12] Gu Xiaoyan et al. Effect of mindfulness training on sleep quality of patients with hypertension. Journal of nursing, 2014.29 (05): 27-29

[13] Zhang Jiwei, Gao Shanshan and Hao Na, application of mindfulness training in elderly patients with hypertension and insomnia. Chinese Journal of convalescent medicine, 2016.25 (03): 257-258

[14] Liu Zhihui, puerarin and mindfulness meditation in the treatment of hypertension with anxiety and depression. Journal of Mathematical Medicine, 2019.32 (07): 1055-1056

[15] Miao Su, Deng Xiaolan and Qian Lei, influence of mindfulness intervention on anxiety and depression level of elderly patients with hypertension. Nursing practice and research, 2017.14 (10): 139-140

[16] Qin Bing and song Liping, effect of mindfulness decompression combined with acupoint massage on anxiety state of patients with essential hypertension, China health nutrition, 2016.26 (33): 400-400401

[17] Goldin, P.R. and J.J. Gross, Effects of mindfulness-based stress reduction (MBSR) on emotion regulation in social anxiety disorder. Emotion, 2010. 10(1): p. 83.

[18] Xu Wei, Wang Yuzheng and Liu Xinghua, the effect of 8-week mindfulness training on negative emotions. Chinese Journal of mental health, 2015.29 (07): 497-502 